\title{
ASOCIACIÓN DE MIELOPEROXIDASA SÉRICA CON VARIABLES CARDIOMETABÓLICAS EN DOS POBLACIONES: CARHUAMAYO (4100 m - JUNÍN) Y MALA (30 m - LIMA)
}

\author{
María R Carreño ${ }^{1}$, Elena R. Benavides ${ }^{1}$, Carmen G. Peña ${ }^{1}$, Alejandro Florentini², \\ Yadira Fernández ${ }^{1}$, Cynthia G. Esquerre ${ }^{1}$, Luz K. Hernández ${ }^{1}$ y Elizabeth Carranza ${ }^{3}$
}

\section{RESUMEN}

El objetivo del presente trabajo fue determinar las concentraciones séricas de la mieloperoxidasa (MPO), una enzima considerada como indicadora de inflamación, en una población de las grandes alturas y en otra del nivel del mar y establecer la asociación de esta enzima con los factores de riesgo tendientes a desarrollar una enfermedad cardiometabólica. Se recolectó muestras de sangre de 65 personas oriundas de la ciudad de Carhuamayo (4100 m - Junín) y de 42 personas de Mala (30 m - Lima). Se midió peso, talla y presión arterial. Se determinó hemoglobina $(\mathrm{Hb})$ en sangre total; glucosa, colesterol total, colesterol de lipoproteínas de alta densidad, triglicéridos y MPO se determinaron en suero. Los resultados muestran que a excepción del peso, talla y $\mathrm{Hb}$ no hay diferencia significativa por género entre los promedios de las demás variables de ambos grupos. Al comparar ambas poblaciones, se encontró diferencia significativa en peso $(<0,001)$, IMC $(\mathrm{p}<0,001), \mathrm{Hb}(\mathrm{p}<0,001)$, glucosa $(<0,001)$ y MPO $(<0,01)$. Los niveles de Hb fueron mayores en la población de Carhuamayo, mientras que el peso, IMC, nivel de glucosa y MPO fueron significativamente menores que los del nivel del mar. Se observó correlación significativa directa entre MPO y peso, cintura, IMC y glucosa. Se concluye que la mieloperoxidasa (MPO) no sería un factor de riesgo de enfermedades cardiometabólicas en poblaciones andinas.

Palabras clave: Mieloperoxidasa, factor de riesgo cardiometabólico, altitud.

\section{SERIC MYELOPEROXIDASE ASSOCIATION WITH CARDIOME- TABOLIC VARIABLES IN TWO POPULATIONS: CARHUAMAYO (4100 M - JUNIN) AND MALA (30 M - LIMA)}

\section{SUMMARY}

The aim of this study was to determine serum myeloperoxidase (MPO) level, an enzyme considered as an inflammation indicator, in a high altitude population and in another at sea level, and establish the association of this enzyme with risk factors apt to develop a

\footnotetext{
${ }^{1}$ Facultad de Farmacia y Bioquímica, UNMSM,

${ }^{2}$ Facultad de Ciencias Biológicas, UNMSM,

${ }^{3}$ Instituto Nacional de Biología Andina, Facultad de Medicina, UNMSM e-mail: mcarrenoq@unmsm.edu.pe
} 
cardiometabolic disease. Blood samples were collected from 42 subjects in Mala (30 m Lima) and from 65 people native to Carhuamayo (Junin $4100 \mathrm{~m}$ ). Weight, height and blood pressure were measured. Hemoglobin $(\mathrm{Hb})$ was determined in whole blood; glucose, total cholesterol, high density lipoproteins, triglycerides and MPO were determined in serum. The results show that with the exception of weight, height and $\mathrm{Hb}$, no significant gender differences between the averages of other variables in both groups were observed. When comparing both populations, highly significant differences in weight $(<0,001)$, $\mathrm{BMI}(<0,001), \mathrm{Hb}(<0,001)$, glucose $(<0,001)$ and MPO $(<0,01)$ were found. Hb levels were higher in the population of Carhuamayo, while the weight, BMI, glucose and MPO were significantly lower than the sea level means. Positive significant correlation between MPO and weight, waist, BMI and glucose was observed. It was concluded that the myeloperoxidase (MPO) would not be a risk factor for cardiometabolic disease in Andean populations.

Key words: Myeloperoxidase, cardiometabolic risk factor, high altitude.

\section{INTRODUCCIÓN}

La mieloperoxidasa (MPO, EC 1.11.1.7) es una hemoproteína que se encuentra almacenada en los neutrófilos activados, monocitos y macrófagos. Es una enzima ligada a la inflamación y al estrés oxidativo; en presencia de iones de cloruro y peróxido de hidrógeno cataliza la formación de ácido hipocloroso, que es un agente microbicida potente; por lo tanto desempeña un papel importante en la defensa del huésped contra los microorganismos ${ }^{1}$.

La MPO también utiliza al $\mathrm{H}_{2} \mathrm{O}_{2}$ y diferentes sustratos endógenos como aminoácidos, nitritos, estradiol, etc., como co-sustratos para generar una amplia gama de especies oxidantes, así se tiene las cloraminas, radical hidroxilo, oxígeno singlete, ozono, radical tirosilo, dióxido de nitrógeno ${ }^{2}$.

Diversos estudios epidemiológicos y clínicos han demostrado que concentraciones altas de MPO se asocian con un mayor riesgo de enfermedades cardiovasculares (ECV), independientemente de los factores de riesgo de ECV clásica ${ }^{3}$.

Se ha demostrado que la MPO es responsable de la conversión de la LDL-nativa a su forma oxidada (LDL-ox), que son reconocidas por los receptores scavengero 'basureros' de los macrófagos, que los internalizan y transforman en células espumosas que conducen a la formación de la placa de ateroma ${ }^{4}$. La MPO puede oxidar la Apo AI de las HDL y alterar su metabolismo, y la consecuencia es que las HDL pierden sus propiedades anti-oxidantes y anti-inflamatorias, y también bloquea la capacidad de la lipoproteína para eliminar el exceso de colesterol de las células ${ }^{4}$.

Por otro lado, se conoce que la MPO puede utilizar al óxido nítrico (NO) como uno de sus sustratos, reduciendo su biodisponibilidad y disminuyendo sus propiedades vasodilatadoras y antiinflamatorias, de esta manera contribuye a la disfunción endotelial, primera etapa del proceso ateroesclerótico ${ }^{3}$. 
Estudios recientes han demostrado que niveles altos de mieloperoxidasa en individuos aparentemente sanos pueden predecir un riesgo futuro de ECV y que la actividad inflamatoria precede por lo menos casi una década a la aparición de $\mathrm{ECV}^{5}$.

Diversos estudios fisiológicos, clínicos y epidemiológicos realizados muestran que existe menor incidencia de enfermedades coronarias en los residentes de altura a diferencia de aquellos de nivel del mar ${ }^{6}$. De los factores de riesgo relacionados es conocido que en los habitantes de las grandes alturas (GA), los niveles de colesterol es menor y los niveles de HDL-colesterol (HDL-col) son mayores que a nivel del mar; es menor la prevalencia de hipertensión arterial, y que el diabético de altura presenta menor riesgo de presentar enfermedad cerebro-vascular que el diabético de nivel del mar, debido a las menores alteraciones lipídicas que presenta ${ }^{6}$.

En el presente estudio se propone determinar la concentración de mieloperoxidasa sérica, su distribución y asociación con varios factores de riesgo relacionados con enfermedades cardiometabólicas en una población de nivel del mar y otra de la altura.

\section{MATERIALES Y MÉTODOS}

\section{Sujetos de experiencia}

Esta investigación es un estudio transversal en el que se incluyeron a 107 personas adultas, 65 (11 H/54 M) procedentes de la ciudad de Carhuamayo (Región Junín, 4100 msnm) y 42 $(12 \mathrm{H} / 30 \mathrm{M})$, naturales de la ciudad de Mala $(30 \mathrm{~m})$. Ninguno de los voluntarios presentaba diabetes mellitus ni enfermedad cardiovascular.

Para la toma de muestras, los voluntarios se presentaron en condiciones de ayuno absoluto (12 horas previas) y sin haber realizado actividad física.

\section{Recolección de las muestras}

Las muestras de sangre, $8 \mathrm{~mL}$ fueron obtenidas por punción venosa del antebrazo y recibidas en tubos de ensayo limpios y esterilizados. La sangre extraída se dejó en reposo por 30 minutos hasta coagulación y luego se centrifugó a $4000 \mathrm{rpm}$, por 5 minutos se separaron los sueros. Se colocaron los sueros entre 2 y $5^{\circ} \mathrm{C}$ en un recipiente hermético, para ser transportados al Laboratorio de Bioquímica del Instituto y almacenados a $-20^{\circ} \mathrm{C}$ para los análisis posteriores.

\section{Mediciones}

La medición de la presión arterial fue realizada por un profesional de la salud en el brazo izquierdo, con la persona sentada; además se pesaron y tallaron a los individuos sin calzado y con ropa ligera; asimismo, se midieron los perímetros de cintura y cadera.

\section{Determinaciones}

Se utilizaron kits comerciales para las determinaciones del nivel de hemoglobina $(\mathrm{Hb})$ en sangre total (Kit de Hemoglobina de VALTEK S.A., Santiago-Chile) y los niveles séricos 
de glucosa (Kit Glucosa - LS (GOD -PAP) de VALTEK S.A., Santiago-Chile), colesterol total (Kit Colesterol CHOD-PAP de VALTEK S.A., Santiago-Chile), HDL-col (Kit HDL (Fosfotungstato/ $\mathrm{Mg}^{++}$) de VALTEK S.A., Santiago-Chile) y triglicéridos (Kit Triglicéridos de VALTEK S.A., Santiago-Chile). La concentración de LDL-colesterol (LDL-col) se determinó aplicando la fórmula de Friedewald ${ }^{7}$. El nivel sérico de mieloperoxidasa se determinó por el método de ELISA utilizando un kit comercial marca MyBioSource, INC. (USA). De acuerdo a normas internacionales, el índice de masa corporal (IMC, peso en $\mathrm{kg}$, dividido entre el cuadrado de la estatura, en metros) entre $25 \leq \mathrm{IMC}<30 \mathrm{~kg} / \mathrm{m}^{2}$ fue considerado como criterio de sobrepeso y el IMC $>30 \mathrm{~kg} / \mathrm{m}^{2}$, como criterio de obesidad ${ }^{7}$.

\section{Análisis estadístico}

Los resultados fueron expresados en valores medios y desviación estándar. Se compararon las medias según la prueba $t$ de Student para dos muestras independientes, y se evaluó el grado de asociación mediante el coeficiente de correlación de Pearson. Se consideró significativo todo resultado cuyo valor asociado de $\mathrm{p}$ sea $<0,05$.

\section{RESULTADOS}

La tabla 1 muestra las características de la población participante. La media de edad de la población de Carhuamayo fue 44,1 $\pm 13,0$ años y de la población de Mala fue 44,2 $\pm 13,6$ años, sin diferencia significativa $(\mathrm{p}>0,05)$. No se encontraron diferencias significativas en cintura, presión sistólica y presión diastólica; se encontró diferencia estadísticamente significativa en cadera $(\mathrm{p}<0,05)$ entre ambos grupos. Los promedios de los pesos de ambas poblaciones, $60,9 \pm 8,9 \mathrm{~kg}$ en Carhuamayo y 72,1 $\pm 13,9 \mathrm{~kg}$ en la población de Mala, presentan diferencia estadística $(\mathrm{p}<0,001)$.

El IMC sigue el mismo patrón por lo que se presenta diferencia estadística $(p<0,001)$ entre ambas poblaciones. 
Tabla 1. Características de la población de estudio altitud

\begin{tabular}{lccc}
\hline & $\begin{array}{c}\text { Carhuamayo } \\
\text { (Media } \pm \mathrm{DE})\end{array}$ & $\begin{array}{c}\text { Mala } \\
(\text { Media } \pm \mathrm{DE})\end{array}$ & $\mathbf{p}$ \\
\hline $\mathrm{n}$ & 65 & 42 & \\
\hline Edad, años & $44,1 \pm 13,0$ & $44,2 \pm 13,6$ & $>0,05$ \\
Peso, kg & $60,9 \pm 8,9$ & $72,1 \pm 13,9$ & $<0,001^{*}$ \\
Talla, m & $1,54 \pm 0,1$ & $1,56 \pm 0,1$ & $>0,05$ \\
IMC, kg/m & $25,6 \pm 3,3$ & $29,2 \pm 4,6$ & $<0,001^{*}$ \\
Cintura, cm & $92,7 \pm 7,0$ & $92,5 \pm 10,4$ & $>0,05$ \\
Cadera, cm & $98,2 \pm 5,0$ & $101,4 \pm 9,3$ & $<0,05 *$ \\
Presión sistólica, mmHg & $11,9 \pm 1,7$ & $12,2 \pm 3,0$ & $>0,05$ \\
Presión diastólica, $\mathrm{mmHg}$ & $7,9 \pm 0,9$ & $7,5 \pm 1,6$ & $>0,05$ \\
\hline
\end{tabular}

*Estadísticamente significativo (t de Student para medias)

Los resultados de hemoglobina y demás parámetros bioquímicos se presentan en la tabla 2. Los niveles de hemoglobina en la población de Carhuamayo $(16,6 \pm 0,6 \mathrm{~g} / \mathrm{dL})$ y en la población de Mala $(13,4 \pm 1,2 \mathrm{~g} / \mathrm{dL})$ difieren estadísticamente, $(\mathrm{p}<0,001)$, igual diferencia significativa se encontró entre los niveles de glucosa sérica de ambas poblaciones $(74,7 \pm$ $12,9 \mathrm{mg} / \mathrm{dL}$ vs $101,6 \pm 26,5, \mathrm{p}<0,001)$ No se encontraron diferencias significativas en cuanto a niveles séricos de los parámetros lipídicos estudiados.

Tabla 2. Variables bioquímicas de la población de estudio

\begin{tabular}{lccc}
\hline & $\begin{array}{c}\text { Carhuamayo } \\
\text { (Media } \pm \mathrm{DE})\end{array}$ & $\begin{array}{c}\text { Mala } \\
(\text { Media } \pm \mathrm{DE})\end{array}$ & $\mathbf{p}$ \\
\hline $\mathrm{n}$ & 65 & 42 & \\
\hline Hemoglobina, g/dL & $16,6 \pm 0,6$ & $13,4 \pm 1,2$ & $<0,001^{*}$ \\
Glucosa, mg/dL & $74,7 \pm 12,9$ & $101,6 \pm 26,5$ & $<0,001^{*}$ \\
Colesterol, mg/dL & $209,2 \pm 48,7$ & $211,6 \pm 34,3$ & $>0,05$ \\
HDL-colesterol, mg/dL & $57,1 \pm 19,5$ & $54,2 \pm 14,3$ & $>0,05$ \\
LDL-colesterol, mg/dL & $116,1 \pm 44,1$ & $118,5 \pm 30,8$ & $>0,05$ \\
\hline Triglicéridos, $\mathrm{mg} / \mathrm{dL}$ & $167,7 \pm 89,3$ & $194,7 \pm 91,0$ & $>0,05$ \\
\hline
\end{tabular}

*Estadísticamente significativo (t de Student para medias) 
La comparación de los valores medios de mieloperoxidasa: $12,9 \pm 9,4 \eta \mathrm{g} / \mathrm{mL}$ para la población de Carhuamayo, y 19,1 $\pm 7,5 \eta \mathrm{g} / \mathrm{mL}$ para la población de Mala, dio como resultado una diferencia significativa entre ambos grupos, $(\mathrm{p}<0,001)$, (figura 1).

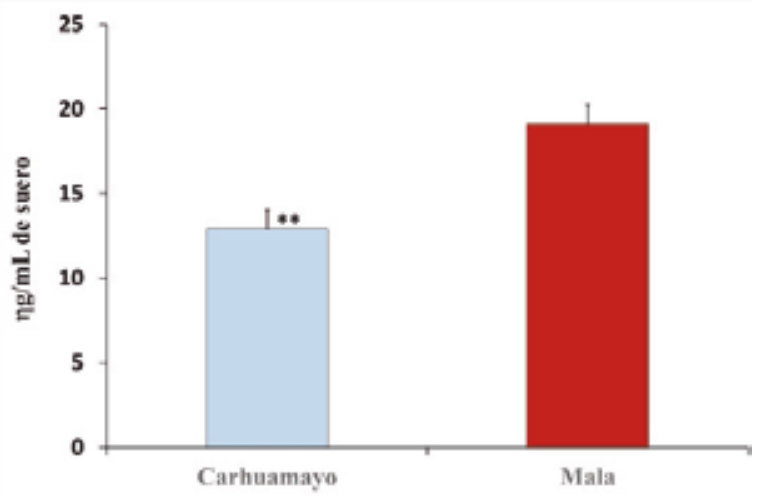

Figura 1. Media \pm Error Estándar de la Media de mieloperoxidasa ( $\eta \mathrm{g} / \mathrm{mL}$ de suero), $* * \mathrm{p}<0,001$ con respecto a la media del grupo del NM

Cuando se analizaron los resultados de la mieloperoxidasa según género, se encontró que el promedio sérico en la totalidad de la mujeres $(\mathrm{n}=84)$ y de los hombres $(\mathrm{n}=23)$ no presentaban diferencia estadística: $14,6 \pm 9,6$ vs $16,5 \pm 8,3$ ( $p>0,05)$, en cambio las diferencias según altitud son significativas en ambos sexos (tabla 3 )

Tabla 3. Niveles séricos de mieloperoxidasa según altitud

\begin{tabular}{lccccc}
\hline & \multicolumn{3}{c}{ Carhuamayo } & & Mala \\
& $\mathrm{n}$ & $($ Media $\pm \mathrm{DE})$ & $\mathrm{n}$ & $($ Media $\pm \mathrm{DE})$ & $\mathbf{p}$ \\
\hline Hombres & 11 & $12,6 \pm 6,9$ & 12 & $20,2 \pm 8,1$ & $<0,05^{*}$ \\
Mujeres & 54 & $13,0 \pm 9,8$ & 30 & $19,0 \pm 7,43$ & $<0,05^{*}$ \\
\hline \multicolumn{4}{r}{ *Estadísticamente significativo (t de Student para medias) } \\
\end{tabular}

Cuando se evaluó el grado de asociación entre el nivel de mieloperoxidasa sérica y las demás variables observadas (tabla 4) se encontró una estrecha relación directa entre niveles séricos de MPO y las variables peso $(\mathrm{p}<0,001)$; cintura $(\mathrm{p}<0,05)$, IMC $(\mathrm{p}<0,001)$ y glucosa $(\mathrm{p}$ $<0,05)$. No se encontró asociación significativa entre el nivel de MPO sérica con los demás parámetros evaluados. 
Tabla 4. Correlación entre nivel de mieloperoxidasa sérica y variables de riesgo cardiometabólico en la muestra total (Carhuamayo y Mala, $\mathrm{n}=107$ )

\begin{tabular}{lcl}
\hline \multicolumn{1}{c}{ Variable } & r & p \\
\hline Edad, años & 0,143 & $>0,05$ \\
Peso, kg & 0,481 & $<0,001^{*}$ \\
Talla, $\mathrm{m}$ & 0,180 & $>0,05$ \\
\hline IMC, kg/m ${ }^{2}$ & 0,376 & $<0,001^{*}$ \\
\hline Cintura, cm & 0,3867 & $<0,05^{*}$ \\
\hline Cadera, cm & 0,307 & $>0,05$ \\
\hline Presión sistólica, mmHg & 0,152 & $>0,05$ \\
\hline Presión diastólica, mmHg & 0,046 & $>0,05$ \\
\hline Glucosa, mg/dL & 0,268 & $<0,05^{*}$ \\
\hline Colesterol, mg/dL & 0,156 & $>0,05$ \\
\hline HDL-colesterol, mg/dL & $-0,041$ & $>0,05$ \\
\hline LDL-colesterol, mg/dL & 0,116 & $>0,05$ \\
\hline Triglicéridos, mg/dL & 0,115 & $>0,05$ \\
\hline \multicolumn{1}{c}{ * Estadisticamente significativo (r de Pearson) }
\end{tabular}

\section{DISCUSIÓN}

Diversos estudios han demostrado que el nivel sérico de MPO está incrementado en la diabetes $^{8}$, hipertension ${ }^{9}$, obesidad ${ }^{10}$, aterosclerosis ${ }^{11}$, etc. Se ha demostrado que los niveles séricos de mieloperoxidasa se asocian con el riesgo de enfermedad cardiovascular en individuos aparentemente $\operatorname{sanos}^{5}$.

En nuestro país, el presente estudio es el primero en presentar niveles séricos de MPO en individuos aparentemente sanos de dos poblaciones, una a nivel del mar y otra en la altura con el fin de establacer si hay diferencias entra ambas.

Se realizó un estudio transversal en una muestra de sujetos oriundos de las grandes alturas (Carhuamayo, $4161 \mathrm{~m}$ ) y otra de la ciudad de Mala $(30 \mathrm{~m})$ para determinar la relación entre los niveles de mieloperoxidasa sérica y algunas variables consideradas como factores de riesgo de enfermedades cardiometabólicas. Así, se encontró que la población de Mala, en promedio, presentó mayor peso e IMC que el grupo de altura; en cambio, la presión arterial y la circunferencia de cintura y de cadera fueron similares en ambos grupos y están dentro de los parámetros establecidos como normales? 
Los niveles de $\mathrm{Hb}$ fueron mayores en el grupo de altura que en el grupo del nivel del mar; el incremento de $\mathrm{Hb}$ en residentes de la grandes alturas es uno de los principales mecanismos de adaptación a la hipoxia de estas altitudes, junto con el incremento en el número de hematíes, y en el hematocrito ${ }^{12}$.

Los niveles de glucemia del grupo de altura fueron significativamente menores que del grupo del nivel del mar, este resultado es similar a lo encontrado por numerosos estudios que han demostrado que en los residentes en la altura, los valores medios de glucosa sérica normalmente son menores que sus similares del nivel mar $^{12}$.

Los resultados de perfil lipídico, obtenidos en este estudio, indican que los valores séricos de colesterol total, LDL-col y HDL-col estaban dentro del rango considerado deseable ${ }^{7}$ para ambas poblaciones y eran similares a los encontrados por otros investigadores ${ }^{13,14}$. Los niveles séricos de triglicéridos (TG) de ambas poblaciones están dentro de límites considerados como "riesgo moderado"; sin embargo difieren de los referidos por otros autores, quienes encontraron valores altos de TG en otras poblaciones andinas ${ }^{15}$.

Los resultados del presente trabajo demuestran que los valores medios de mieloperoxidasa en el grupo de Mala son significativamente más altos $(p<0,001)$ que los correspondientes de Carhuamayo; vale decir que la población de la altura estaría mejor protegida frente a la inflamación, lo que significaría una menor producción de la enzima $y$, por ende, un menor efecto adverso de la MPO, pues se conoce que la generación excesiva de oxidantes por MPO está relacionada con el daño tisular en muchas enfermedades, especialmente las caracterizadas por inflamación aguda o crónica ${ }^{16}$. No se encontraron diferencias significativas entre hombres y mujeres.

Los mecanismos por los cuales en la altura hay un menor nivel sérico de esta enzima, no se conocen; es necesario más estudios en otros grupos etarios, tanto de nivel del mar como de altura, para poder comprender el papel de la MPO en la prevención de las enfermedades cardiovasculares en poblaciones que viven en las grandes alturas ${ }^{6}$.

La relacion directa y significativa entre nivel de MPO y peso y cintura y glucosa, confirma lo hallado por otros autores, es decir que el nivel sérico de MPO está incrementado en casos de obesidad ${ }^{10} \mathrm{y}$ de diabetes ${ }^{8}$. Se ha demostrado una relación entre obesidad e inflamación ${ }^{17}$ y se ha encontrado también que niveles altos de glucosa puede promover una reacción inflamatoria, lo que lleva a un aumento de los niveles de MPO en plasma ${ }^{18}$.

\section{CONCLUSIONES}

Los resultados del presente trabajo sugieren que la mieloperoxidasa (MPO) no sería un factor de riesgo de enfermedades cardiometabólicas en habitantes de las grandes alturas y que los niveles menores encontrados de esta enzima estarían relacionados con los menores promedios de peso y nivel de glucosa hallados en este grupo. 


\section{AGRADECIMIENTO}

Este trabajo fue financiado por el Proyecto del VRI N. ${ }^{\circ} 140403051$.

\section{REFERENCIAS BIBLIOGRÁFICAS}

1. Klebanoff SJ. Myeloperoxidase: friend and foe. J Leukoc Biol 2005;77:598-625.

2. Davies MJ, Hawkins CL, Pattison DI, Rees MD. Mammalian heme peroxidases: from molecular mechanisms to health implications. Antioxid Redox Signal. 2008; 10:11991234.

3. Brennan ML, Hazen SL. Myeloperoxidase: A Mechanistically Linked Biomarker for Cardiovascular Disease. Curr Cardiovasc Risk Rep. 2007; 1:58-65

4. Nicholls SJ, Hazen SL. Myeloperoxidase, modified lipoproteins and atherogenesis. J Lipid Res. 2009; 50 (suppl): S346-51.

5. Meuwese MC, Stroes ES, Hazen SL, van Miert JN, Kuivenhoven JA, Schaub, et al. Serum myeloperoxidase levels are associated with the future risk of coronary artery disease in apparently healthy individuals: the EPIC-Norfolk Prospective Population Study. J Am Coll Cardiol. 2007; 50(2): 159-65.

6. Gonzales G. Metabolismo en las grandes alturas. Acta Andina. 2001; 9: 31-42

7. National Cholesterol Education Program (NCEP). Third Report of the National Cholesterol Education Program (NCEP) Expert Panel on Detection, Evaluation, and Treatment of High Blood Cholesterol In Adults (Adult Treatment Panel III) final report. Circulation. 2002; 106:3143-3421.

8. Wiersma JJ, Meuwese MC, van Miert JN, Kastelein A, Tijssen JG, Piek JJ, et al. Diabetes mellitus type 2 is associated with higher levels of myeloperoxidase. Med Sci Monit. 2008; 14: 406-10.

9. Van der Zwan LP, Scheffer PG, Dekker JM, Stehouwer CD, Heine RJ,Teerlink T. Hyperglycemia and oxidative stress strengthen the association between myeloperoxidase and blood pressure. Hypertension. 2010;55: 1366-1372.

10. Olza J, Aguilera CM, Gil-Campos M, Leis R, Bueno G, Martínez-Jimenez MD, et al. Myeloperoxidase is an early biomarker of inflammation and cardiovascular risk in prepubertal obese children. Diabetes Care. 2012; 35:2373-2376.

11. Baldus S, Heeschen C, Meinertz T, Zeiher AM, Eiserich JP, Munzel T, et al. Myeloperoxidase serum levels predict risk in patients with acute coronary syndromes. Circulation. 2003;108: 1440-5.

12. West JB, Schoene RB, Milledge JS. High altitude medicine and physiology. $4^{\text {th }}$ ed. London: Hodder Arnold a Member of the Hodder Headline Group; 2007.

13. Pajuelo J, Sánchez-Abanto J, Torres H, Miranda M. Prevalencia del síndrome metabólico en pobladores peruanos por debajo de 1000 y por encima de los $3000 \mathrm{msnm}$. An Fac Med. 2012; 73(2):101-106.

14. Mohanna S, Baracco R. Seclen S. Lipid profile, waist circumference, and body mass index in a high altitude population. High Alt Med Biol. 2006; 7:245-55.

15. Gonzáles GF, Tapía V. Asociación de los diferentes niveles de hipoxemia en la altura 
con el perfil lipídico y la glucemia en varones y mujeres a 4,100 m de altitud en los Andes Centrales del Perú. Endocrinol Nutr. 2012; 60:79-86.

16. Van der Veen BS, de Winther MP, Heeringa P. Myeloperoxidase: molecular mechanisms of action and their relevance to human health and disease. Antioxid Redox Signal. 2009; 11:2899-2937

17. Gregor MF, Hotamisligil GS. Inflammatory mechanisms in obesity. Ann Rev Immunol. $2011 ; 29: 415-45$.

18. Zhang X, Dong L, Wang Q, Xie X. The relationship between fasting plasma glucose and MPO in patients with acute coronary syndrome. BMC Cardiovasc Disord. 2015; 15:1-7. 